\title{
Oferta de nuevas viviendas y factores institucionales
}

\author{
Paloma Taltavull de La Paz \\ Universidad de Alicante
}

\section{Resumen}

Este artículo aborda la oferta de vivienda nueva en España y el efecto del aumento en los solares disponibles para construcción resultantes de la aplicación de los planes de vivienda. La literatura existente respalda la fuerte reacción de los promotores (españoles) ante cambios en los precios residenciales, siguiendo los principios de mercado. Este artículo utiliza información secundaria y estima un modelo de oferta de vivienda nueva con precios, costes y añade el papel de la oferta de suelo con el objeto de individualizar su efecto del resto de las particularidades geográficas. El modelo es calculado utilizando métodos de panel a nivel de regiones. Los resultados muestran que solo seis de las CCAA analizadas registran elasticidades estadísticamente significativas de la oferta de suelo, interpretándose de este resultado que son fuertemente sensibles a los procesos institucionales para su creación. Las elevadas elasticidades precio-oferta en España, salvo en Madrid, explicaría el ajuste drástico a la baja de los ciclos de edificación tras la crisis de 2007.

Palabras clave: oferta de viviendas, mercado residencial en España, restricción crediticia, elasticidad.

Clasificación JEL: R31.

\begin{abstract}
The paper approaches the new housing supply model and the effect of land availability in. It adds evidence from the Spanish housing market to the current literature on housing supply. Using secondary data, the paper estimates a model of new supply which includes price, cost of construction materials, cost of labour elasticity and available land to build measured by number of urban slots. Models are calculated using panel methodology for the whole Spain and its regions (nut 2 level). Results show how land elasticity are only statistically significant in six out of sixteen Spanish regions which can be interpreted as a strong sensibility to the institutional process of land creation in those communities. The empirical results suggest that the strong price sensibility shown by larger elasticities could explain the quick fall in starts cycles among the whole Spanish territory.
\end{abstract}

Keywords: housing supply, Spanish housing market, credit crunch, elasticity.

JEL classification: R31.

\section{Introducción}

La crisis financiera inició una fuerte caída en la construcción de viviendas en la mayoría de los países desarrollados desde 2007. Después de un largo periodo de revalorización y edificación (con solo unas pocas excepciones), la crisis financiera global detuvo el ciclo de la vivienda de repente y con un ajuste de los precios residenciales más lenta que en otros periodos previos. Lo duro de la contracción se refleja en la caída de la edificación residencial en España, la mayor de los países 
europeos, donde la construcción de viviendas había superado el máximo histórico de actividad durante la década de 2000 para caer repentinamente desde 2008 y alcanzar el mínimo histórico (desde la década de los sesenta) en tan solo un año y medio. Esto supuso un estancamiento, literalmente, en la industria de construcción de viviendas y una rémora al crecimiento económico desde ese momento.

Aunque el número de viviendas iniciadas durante la primera década del siglo XXI parecía estar desequilibrada con las necesidades tradicionales de la población española, lo cierto es que los fuertes cambios en las fuentes de demanda que se produjeron, ejercieron presión en el mercado para aumentar la oferta. Estos cambios abarcan desde un flujo neto de inmigrantes de más de 4 millones de personas en tan solo siete años (2000-2007), los cambios en los patrones de movilidad de la población nacional, así como un incremento desconocido anteriormente en la demanda residencial de los ciudadanos europeos en algunas regiones españolas. Estas nuevas fuentes de demanda demográfica añadidas a las procedentes del crecimiento doméstico o privado, han, probablemente, cambiado el patrón de demanda que afecta especialmente a los mecanismos del mercado de la vivienda en España. En el Gráfico 1 se puede ver cómo se produce el cambio en el patrón de migración (movilidad entre las regiones), así como la adición de un flujo neto positivo sistemático de hogares a la demanda de viviendas (inmigración). Esta fuerte demanda se considera que ha

\section{GRÁFICO 1}

MOVILIDAD DE LA POBLACIÓN. ALTAS Y BAJAS RESIDENCIALES

(En medias móviles M1212 sobre la serie original)

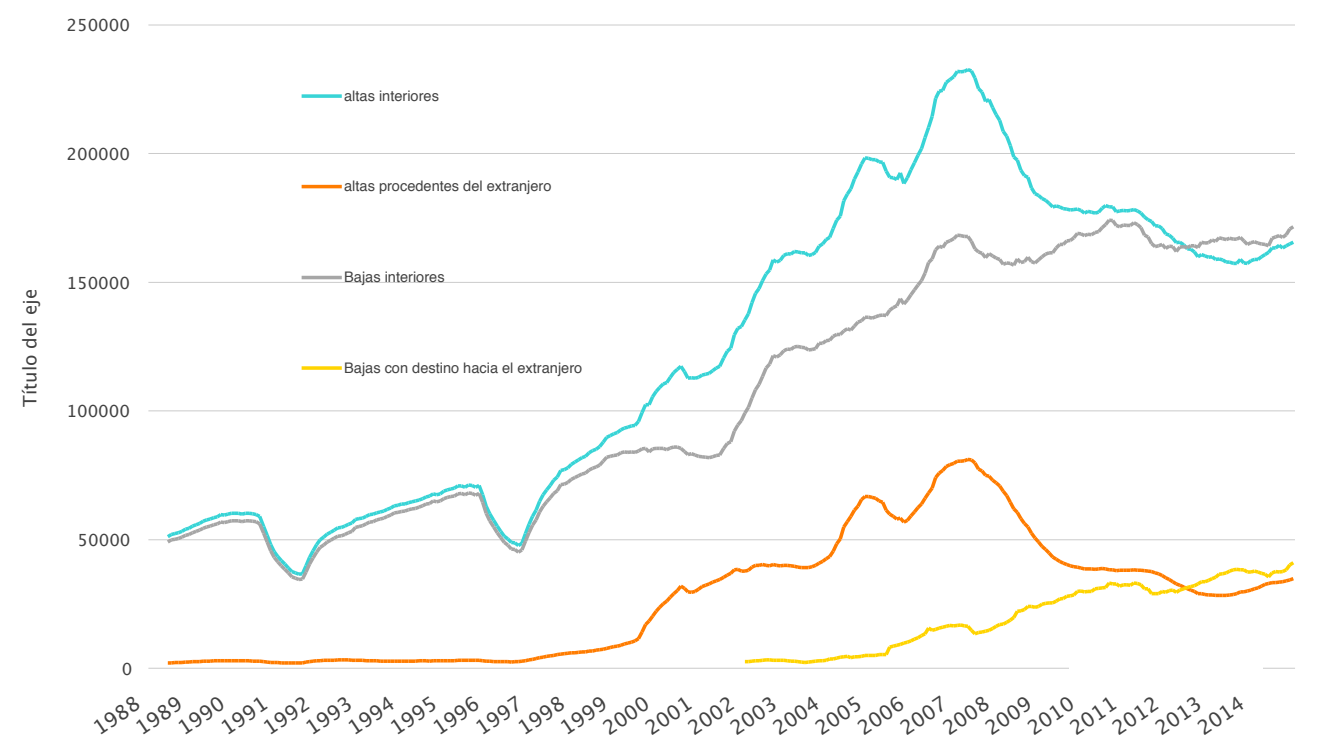

FUENTE: EVR, INE. 
actuado de incentivo de la edificación residencial en España hasta alcanzar unas tasas de crecimiento mayores de lo habitual, lo que pudo dar la imagen mencionada de construcción desordenada.

Este no es un hecho único en España. La literatura internacional contrasta cómo la construcción más intensa se correlaciona con las regiones que reciben más población, básicamente concentrándose en las principales ciudades, áreas de actividad económica, por razones laborales, o zonas estrictamente residenciales. Esto parece haber ocurrido en distintas regiones del mundo, como en Nueva Zelanda (Grimes, 2007). Es un hecho estilizado que la mayoría de regiones con similares características están relacionados con el flujo permanente de la migración con fines residenciales (Gibler et al., 2009) y confirman un aumento de la población demandante que, en ocasiones, no es observado a través de las estadísticas de forma directa.

En España, los ciclos de edificación alcanzan su máximo justo antes de la crisis financiera global, con un pico alrededor de 60.000 unidades por mes en el año 2007. La intensidad en la edificación muestra fuertes diferencias a lo largo de las regiones, como se observa en el Gráfico 2, y la contracción posterior parece ser muy similar aunque, por las diferencias, con un mayor impacto en las áreas con actividad previa más intensa.

\section{GRÁFICO 2 \\ CICLOS DE EDIFICACIÓN EN ESPAÑA \\ (En número de licencias de viviendas concedidas para iniciar. Medias Móviles centradas)}

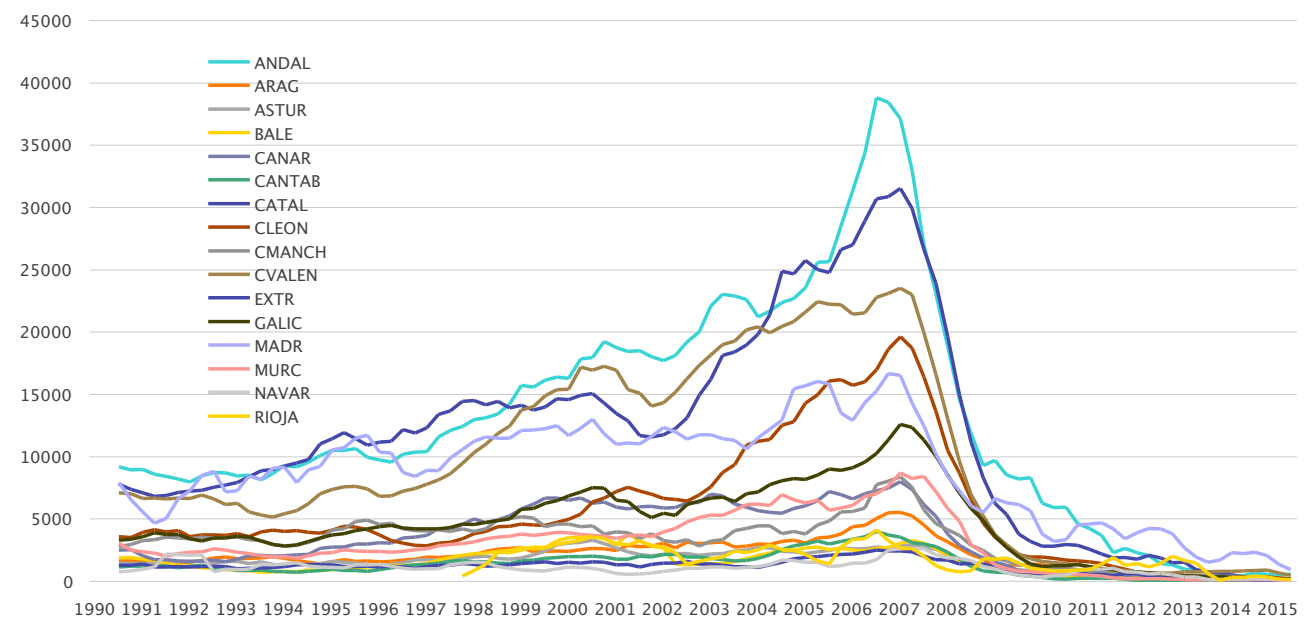

FUENTE: EVR, INE. 
Lo largo de este ciclo debería haber sorprendido a los analistas, ya que tradicionalmente, los límites en la oferta de materiales, mano de obra o el agotamiento de la demanda, deberían haber limitado su duración. Sin embargo, el crecimiento de prácticamente todos los factores de demanda (demográficos arriba vistos, bajos tipos de interés, oferta de crédito casi ilimitada) y de oferta (mano de obra suficiente para la edificación y producción autóctona de materiales), pudieron permitir la continuidad del ciclo expansivo.

La literatura sobre el mercado inmobiliario enseña cómo los cambios en la demanda tienen un efecto inmediato sobre los precios residenciales y estos, a continuación, sobre la edificación (DiPasquale y Wheaton, 1994), lo que implica que la explicación de los ciclos de edificación que se muestran en el Gráfico 2, debe circunscribirse a un entorno de análisis del propio mecanismo de mercado. Hay algunos trabajos que han identificado razones de mercado que explican los ciclos de edificación en España.

Este artículo analiza el mecanismo de la oferta de vivienda nueva en las regiones españolas y aísla los efectos específicos que tiene la oferta de suelo (derivada de la aplicación de los planes de ordenación urbana y de su regulación) y su relación con la respuesta de la edificación ante los incentivos-precio. La hipótesis básica determina que la mayoría de los mercados de vivienda regionales muestran una «reacción del mercado» durante las dos últimas décadas, es decir, que reaccionan a los cambios en la demanda de viviendas sin aparente restricción desde el lado de la oferta. El objetivo del trabajo es evaluar la respuesta de cada región a este incentivo de mercado y cuantificar la existencia de barreras de «no mercado» que han podido condicionar las decisiones de los promotores, como la falta de suelo y otras no observables, como serían el poder de mercadolos derivados del ordenamiento público o de los procedimientos administrativos.

Este trabajo continua otros previos donde se estima la elasticidad de la nueva oferta por regiones (Taltavull, 2006, 2014) y se aporta evidencia empírica sobre la similitud de las reacciones en distintas regiones de España e Italia (Taltavull y Gabrielli, 2015). En ellos se analiza cómo los sectores de oferta nueva de viviendas de las regiones españolas reaccionan al incentivo de mercado (precio) pudiéndose clasificar entre dos grupos: las regiones elásticas, donde la reacción de la construcción ante cambios en los precios es intensa o unitaria, y regiones con muy baja elasticidad o donde este incentivo no es estadísticamente significativo. Las regiones mayores solían clasificarse en el grupo central de elasticidad, aunque alguna de ellas (como la de Madrid) no reflejaba una reacción de mercado. Este patrón se repetía también para el caso de Italia. Los distintos valores de las elasticidades capturaban lo que era un corolario relevante: aquellas regiones con mayores elasticidades, las que veían aumentar más la edificación en referencia a sus precios, reflejaban aumentos en éstos últimos inferiores a los que podrían haber sido en el caso de que sus promotores (o los sistemas institucionales) hubiesen manifestado una reacción más inflexible. Lo contrario ocurría en las regiones donde las elasticidades estimadas eran pequeñas o no había evidencia empírica de relación. 
La comprensión de la distinta capacidad explicativa del mecanismo de mercado descansaba en los condicionantes específicos de la oferta, institucionales, administrativos y de disponibilidad de suelo. En este trabajo se incorpora una medición del suelo disponible para despejar una de las limitaciones a la explicación de la reacción de la oferta que identificaban los trabajos mencionados. Es decir, se «extraen» los efectos de la disponibilidad de suelo del cajón de sastre de los efectos regionales, y se observa cómo actúan en la toma de decisiones de los promotores, aislando en mayor medida las características territoriales (y no observables) que interactúan con el proceso de toma de decisiones de promoción.

El artículo se organiza como sigue. En la sección 2 se revisa la literatura. En la sección 3 se establece el modelo. La sección 4 muestra los datos y la estrategia de estimación. La sección 5 muestra el ejercicio empírico y comenta los resultados, y con la sección 6 se concluye.

\section{Revisión de la literatura}

La oferta de viviendas ha sido analizada en la literatura desde dos ópticas, estudiando el total de la oferta constituido por el stock existente en el mercado (utilizado o vacante) y, en segundo lugar, analizando la evolución de la vivienda nueva. La oferta-stock de viviendas es considerada rígida en el corto plazo (debido a que el proceso de producción es largo, y a la existencia de limitaciones desde los inputs -especialmente suelo- y otros factores) y cambia en el largo plazo como resultado de las características específicas de los mercados de viviendas y de su proceso de producción (Arnott, 1987).

La función de oferta de un mercado es difícil de determinar (Hanusheck y Quigley, 1979), tanto porque la construcción de unidades no es la única fuente de abastecimiento de la oferta total (que se conforma por unidades ya existentes y nuevas, Goodman, 2005), como por la falta de datos disponibles para observar toda la oferta como flujo, y también porque la función de oferta nueva es local y específica de diferentes regiones, en muchos casos, distinta entre las áreas metropolitanas (Glaeser et al., 2005; DiPasquale, 1999).

La oferta de viviendas nuevas (unidades que se añaden al stock) muestra un grado mayor de inflexibilidad en el corto plazo, con reacciones elásticas en el largo plazo que difieren dependiendo del signo del impacto que el sector reciba. La razón de esta asimetría consiste en el propio mecanismo de mercado: En el corto plazo, la construcción de viviendas reacciona al aumento de los precios (consecuencia de un shock en la demanda) con distintos grados de (baja) de sensibilidad dependiendo de las características propias del mercado local (disponibilidad de suelo, regulaciones, estructura empresarial, expectativas) pero aumentando en el medio plazo la oferta (por que el proceso de producción es lento al requerir la movilización del suelo, planes, proceso de construcción (DiPasquale, 1999; Topel y Rosen, 1988). Esta reacción desfasada contribuye a un aumento mayor de los precios. La oferta de 
viviendas nuevas aumenta paso a paso durante un largo periodo, aportando nuevas unidades al mercado ya en momentos en que la demanda declina. Ya en contracción, las unidades en oferta se acumulan en el mercado por falta de demanda, aumentando las vacantes aunque no con una sustancial caída en los precios dado que los costes en los que se ha incurrido y las expectativas de un futuro crecimiento de los valores (en el largo plazo) persuaden a los compradores a mantener las unidades y no venderlas a menor precio. Esta es la razón por la que la respuesta de la oferta de viviendas nuevas es elástica en expansión pero inelástica en contracción (Glaeser et al., 2005), y cuando se estima con ambos periodos, la sensibilidad de la oferta en el largo plazo puede variar, generalmente al alza.

En general se acepta que las elasticidades de oferta nueva a corto plazo son más pequeñas que las de largo plazo porque se requiere un tiempo para la construcción y por las razones mencionadas (Quigley, 1997; Topel y Rosen, 1988; Malpezzi y MacLennan, 2001; Dipasquale y Wheaton, 1994; Goodman, 2005; Malpezzi y Vandell, 2002, entre otros), aunque la evidencia es dispar. Por ejemplo, la investigación de Estados Unidos dan valores mayores de la elasticidad en el largo plazo que en los análisis en países europeos (Meen, 2003; Barker, 2003; Pryce, 1999; Malpezzi y Maclennan, 2001; Bramley, 2003), lo que parece sugerir distintas respuestas desde el lado de la oferta nueva a similares tensiones de demanda, dependiendo de factores que diferencian las economías, en su mayor parte, institucionales (véase Green, Malpezzi y Mayo, 2005) ${ }^{1}$.

La literatura también demuestra que las elasticidades cambian en el tiempo mostrando funciones que tienden a «moverse» (Pryce, 1999; Bramley, 1993, 2003; Malpezzi y Vandel, 2002; Goodman, 2005); con elasticidades cambiantes también en el espacio como resultado de la acción de factores territoriales que afectan a los mercados locales, como el clima (Fergus, 1999) o la localización espacial (Goodman y Thibodeau, 1998). El efecto de la localización espacial y su restricción geográfica se ha analizado por Saiz (2010), donde específicamente se computan la cantidad de suelo disponible para construir en la estimación de las elasticidades utilizando medición georeferenciada.

Aunque sea con desfases, el crecimiento de los ciclos de edificación de nuevas unidades es una reacción deseada dados los fuertes efectos de arrastre que esta actividad tiene en el conjunto de la economía. Es por esta razón por la que el gobierno británico comenzó un proceso profundo de análisis para identificar por qué el Reino Unido no había experimentado el ciclo expansivo de edificación que sí se produjo en un buen número de economías avanzadas (Estados Unidos, Australia, y bastantes europeos como en Holanda, España e Irlanda). El informe del Tesoro del Reino Unido conocido como Barker Review (Barker, 2003) concluía que el ciclo de edificación no había crecido como consecuencias de restricciones institucionales a la oferta, como las administrativas fundamentalmente. El interés en conocer el papel de los distintos determinantes de la oferta de vivienda nueva ha generado múltiples

${ }^{1}$ Para un detalle en profundidad de la literatura sobre elasticidades, véase TALTAVULL (2014). 
contribuciones que aportan explicaciones al mecanismo teórico (Rosenthal, 1999; Mayo y Sheppard, 2001; Malpezzi y McLennan, 2001).

El modelo de oferta de viviendas nuevas que refleja el mecanismo de mercado suele incluir la reacción de los promotores ante los cambios en los precios y, como en una función de producción, en los costes relacionados con el proceso de construcción, discriminando entre costes de materiales, mano de obra y costes financieros (Mayer y Somerville, 2000; Somerville, 1999a; Blackley, 1999; Coulson, 1999), y los costes de suelo (Blackley, 1999), tomando por separado todos ellos ya que difieren dependiendo de las características locales (Goodman, 2005, Malpezzi y Vandel, 2002). La literatura contrasta que las elasticidades de respuesta de los promotores ante la señal de mercado (proceso de toma de decisiones de construcción) pueden diferir intensamente entre mercados y regiones, poniendo el acento en tres grupos de factores: la disponibilidad de suelo, la regulación (tanto el sistema de concesión de permisos como las regulaciones sobre el suelo), y la intervención de la política pública en el mercado. Muchos de estos factores son difícilmente observables, lo que ha generado una amplia literatura que mide estos aspectos y sus efectos para explicar los ciclos de edificación.

En el caso de la regulación, la mayor parte de los trabajos están relacionados con el control del mercado del suelo y los procesos de zonificación, que afectan a la posibilidad de construir, así como las externalidades en los mercados de vivienda y los controles administrativos (Malpezzi, 1996). Los estudios recientes tienden a cuantificar el impacto de los paquetes normativos sobre los precios de las viviendas y su efecto resultante en la oferta de viviendas (Gyourko y Summers, 2006; Gyourko et al., 2008).

La oferta de vivienda es también el resultado de un proceso de toma de decisiones complejas de los promotores (Hanusheck y Quigley, 1979). Hay un acuerdo sobre la aparición de diferentes condiciones en el mercado de la vivienda que promueven la existencia de barreras a la decisión de construir de los promotores, limitando el efecto de los incentivos de mercado y creando un cuasi-monopolio o situaciones de competencia monopolística (Green y Malpezzi, 2003; Quigley, 2007). El poder de mercado que tengan los actores privados o públicos determina el grado de reacción de la oferta de vivienda, pudiendo justificarse las reacciones inelásticas en aquellos casos en que se produzca una concentración de la propiedad de suelo, haya un reducido número de empresas de construcción, o exista un sistema de permisos restrictivos, entre otras razones. Adicionalmente, la decisión de construir depende, también, de los beneficios esperados por el promotor, que se reflejan en el ratio coste/precio, siendo la función de oferta de vivienda nueva el resultado de las fuerzas que dirigen la decisión de inversión en vivienda como activo y siguiendo, por consecuencia, los principios de la $q$ de Tobin (Poterba, 1991).

En definitiva, la literatura muestra que la oferta de nuevas viviendas suele responder a una combinación compleja de incentivos de mercado, dependiendo de la sensibilidad de la decisión empresarial a los cambios, no solo de las señales de mercado (precios y costes), sino también de las de no mercado asociadas a la 
institucional existente en el territorio. Esta estructura institucional se identificaría con la organización del sector empresarial (número de empresas que toman la decisión de construir) que están en relación directa con la disponibilidad de suelo, por un lado, y con el conjunto de regulaciones administrativas existentes (distintas por regiones en España), por otro. La intervención de la política de vivienda es también un factor de incidencia.

Para España, los trabajos previos muestran como las elasticidades de respuesta de la nueva oferta ante cambios en los precios son relevantes y difieren según las regiones, delimitando aquellas que responden a los principios de mercado con otras donde la mayor parte de la reacción descansa en los elementos inobservados. En este artículo se da un paso más, aportando evidencia empírica nueva a través de una medición de la oferta de suelo existente en cada una de las regiones como reflejo de una de las acciones institucionales (la regulación sobre el suelo y el planeamiento) y de la reacción de la oferta a sus cambios durante el periodo, de manera que se identifican, separándolos, los factores regulatorios (no observables) con la disponibilidad de suelo.

\section{El modelo}

Partiendo de Taltavull (2014:10), se define la función de oferta de viviendas nuevas como en [1]:

$$
q_{H s, t}=\alpha_{1}+\alpha_{2} P_{H, t}+\alpha_{3} C m_{t}+\alpha_{4} C s_{t}+\alpha_{5} r i_{t}+\alpha_{6}\left[\eta_{k} G_{t}^{k}\right]+v_{\mathrm{t}}
$$

donde ${ }^{2}$ :

$q_{H s, t}:$ Flujo de viviendas nuevas en oferta.

$P_{H, t}$ : Precio de la vivienda en términos reales.

$\mathrm{Cm}$ : Costes asociados a los materiales de construcción.

$C s_{t}$ : Indicador de coste salarial de los trabajadores de la construcción.

$r i$ : Tasas de interés reales pagados por los promotores.

$\left[\eta_{k} G_{t}{ }^{k}\right.$ : Conjunto de las características del mercado regional, incluyendo características físicas, así como otros aspectos como la oferta de suelo.

$v_{\tau}$ : Término aleatorio.

$\alpha_{1 . .6}$ : Parámetros a estimar.

\footnotetext{
${ }^{2}$ En la definición original, el artículo incluye el factor $\pi_{H}^{e}$ de expectativas de los promotores con respecto a la inflación en viviendas. Posteriormente, este factor es estimado conjuntamente en el modelo por lo que se elimina de la definición.
} 
Dado que el componente de suelo en la definición anterior forma parte del factor específico de la región $\left[\eta_{k} G_{t}{ }^{k}\right.$, este trabajo separa este factor en dos: uno, $S_{i}$ es una variable que aproxima la oferta de suelo y, por tanto, cuantifica la reacción de la oferta nueva de viviendas a la existencia de suelo. El segundo factor es $\mathrm{G}_{\mathrm{t}}^{\mathrm{k}}$, que aproxima la reacción de la nueva oferta ante otros factores institucionales que diferencian las regiones. Así, el modelo a estimar sería:

$$
q_{H s, t}=\beta_{1}+\beta_{2} C m_{t}+\beta_{3} C s_{t}+\beta_{4} P_{H, t}+\beta_{5} r i_{t}+\beta_{6} S_{i, t}+\beta_{7} G_{t}^{k}+\mu_{t}
$$

donde los betas son los parámetros a estimar y $\mu_{t}$ es el término de error. En datos globales, $\beta_{7} G_{t}^{k}$ representa los efectos de componentes inobservados que afectan a la regulación o las características distintivas del mercado de la vivienda, entre ellas la idiosincrasia del sector de la promoción en sus decisiones y los efectos de la política de vivienda, propios de las regiones y diferentes entre ellas.

\section{Los datos y la estrategia de análisis}

Los datos que se utilizan en este artículo se refieren al mercado global español medido a través de sus regiones (Comunidades Autónomas). La idea es identificar el papel de la oferta del suelo en las respuestas de mercado de cada una de ellas, que ayude a entender la evolución del ciclo de edificación y la sensibilidad de la construcción residencial.

Con las series temporales disponibles en fuentes secundarias, se construye un panel de periodicidad trimestral para un periodo de 25 años, desde 1990 hasta 2015 (primer trimestre). Incluye los datos de 16 de las regiones de España (15 comunidades autónomas, sin Navarra y el País Vasco, más la suma de las dos ciudades autónomas, Ceuta y Melilla).

La fuente de los precios residenciales es el Ministerio de Fomento y han sido deflactados utilizando el IPC de cada CCAA. Se mide la oferta nueva a través de las viviendas iniciadas. La fuente de esta variable es el Ministerio de Fomento, que le denomina «permisos municipales para construcción de viviendas». Los costes de construcción se obtienen del Ministerio de Fomento y son agregados a nivel nacional ya que no existen desagregados para cada CCAA, distinguiendo entre los costes asociados a los materiales y los correspondientes a la mano de obra (siguiendo lo recomendado por Coulson, 1999a) dentro del modelo. Los tipos de interés reales se calculan utilizando los tipos de referencia del mercado hipotecario, ajustados por la inflación existente en cada región geográfica, por lo que el panel incorpora las tasas de interés reales asociados a la financiación de la vivienda incluyendo las diferencias de inflación de las regiones españolas. El indicador de disponibilidad de suelo utilizado es el número de parcelas urbanas existentes en la CCAA, dato disponible con base anual en el Catastro. Este indicador incluye tanto las parcelas construidas como aquellas disponibles para construir, y evoluciona con la creación 
legal de nuevas parcelas resultantes de la aplicación de los planes de ordenación urbana en las regiones. Su variación aproxima la disponibilidad de suelo, por lo que es un buen indicador para evaluar el efecto que su oferta tiene para la toma de decisiones de construcción. Esta fuente no publica datos para Navarra y el País Vasco durante el periodo completo, por lo que ambas regiones han sido eliminadas del análisis. El número de parcelas aumenta de forma estable durante el periodo considerado, con las excepciones de Galicia y Asturias, donde este número acelera su crecimiento desde 2003. Las series son utilizadas en términos logarítmicos con el fin de estimar parámetros que reflejen las elasticidades de respuesta. La única variable en niveles del modelo son los tipos de interés reales. Un resumen estadístico de la base se encuentra en el Cuadro 1 del Anexo.

Se aplican técnicas de panel para la estimación del modelo. Dada la naturaleza temporal de las series, se ha seguido la pauta habitual identificando la existencia de raíces unitarias y de cointegración en el panel. Los tests se encuentran en el Cuadro 2 del Anexo. Los resultados sugieren la no existencia de una raíz común en el panel aunque no puede rechazarse (coinciden los tests IPSW, ADF y PP) la existencia de procesos individuales, lo que sugiere la inclusión de procesos autorregresivos por regiones para capturar este efecto estadístico. La inexistencia de relación de cointegración puede ser rechazada igualmente con el respaldo la totalidad de los tests, tanto dentro como entre las regiones (cuadro 3, en el anexo), a la par que respalda la existencia de AR(1) individuales que deben ser integrados en el análisis.

Por último, se ha evaluado la existencia de cambios estructurales en el panel a través del análisis de los residuos resultantes del modelo. Los tests indican la existencia de dos cambios estructurales (2007 y 2010), que afectan a algunas regiones. Se incorporan al análisis, por este resultado, dos variables dummy que corrigen los efectos producidos en ambos años, considerándose cambios de carácter transitorio en cada uno de ellos.

La expresión del modelo final estimado es:

$$
\begin{gathered}
\ln V i v_{i k, t}=\beta_{1}+\beta_{2} \ln C m_{i t}+\beta_{3} \ln C s_{i t}+\beta_{4, k} \ln P_{H i k, t}+\beta_{5, k} r i_{i k, t}+\beta_{6, k} \ln S_{i k, t}+ \\
+\beta_{7, k} G_{k}+\Gamma_{j k}\left(\text { shock }_{j}\right)+\rho_{i k} \mu_{t-1}+\varepsilon_{i t}
\end{gathered}
$$

donde $i$ refleja el orden de las series, $k$ el número de regiones (secciones cruzadas), $t$ es el tiempo y $\rho_{i k} \mu_{t-1}$ representa el proceso autorregresivo de cada región, con $\varepsilon$ como matriz de it errores aleatorios. Al estar medidas las variables en logaritmos, los parámetros obtenidos pueden interpretarse como elasticidades. Los $G_{k}$ son capturados por los efectos fijos por regiones y se incluyen en el modelo dos shocks, el de 2007 y 2010 , con una matriz $\Gamma_{j k}\left(\right.$ shock $\left._{j}\right)$ de los cambios estructurales transitorios significativos en el test de residuos, aproximados con variables dummies que toman valor 1 para las observaciones del año en cuestión, y cero en el resto.

Dado que en España, las regiones son las responsables de aplicar los planes de ordenación del territorio (a propuesta de los municipios) y la distinta regulación 
entre ellas, se espera que parte de ese efecto (en lo que no afecte a la oferta efectiva de suelo) sea capturado por el efecto fijo, mientras que el indicador de oferta de suelo capture en su parámetro la sensibilidad de la edificación hacia éste. Por esta razón, se ajustan dos modelos, el completo y el restringido sin oferta de suelo, y sus parámetros se comparan para respaldar la robustez del modelo.

El modelo completo se estima utilizando mínimos cuadrados en datos de panel incluyendo un estimador de efectos fijos por regiones ${ }^{3}$. Los parámetros obtenidos son consistentes con la teoría y con otros trabajos previos. Los resultados se encuentran en el Cuadro 4 del Anexo.

\section{Resultados e interpretación}

Los resultados del modelo son acordes con la literatura obteniendo parámetros que son estadísticamente significativos en los costes y precios.

Los costes de construcción son significativos en las dos ecuaciones. Los costes de mano de obra están relacionados negativamente con la promoción de nuevas viviendas (como se esperaba ex ante) con un valor muy elevado de la elasticidad, indicando una gran sensibilidad de la construcción hacia ellos (valores superiores a $-7,5$ en ambos casos). Los costes de materiales presentan una sensibilidad positiva. Este resultado coincide con otros en la literatura y se explica porque el parámetro captura cierta endogeneidad entre edificación nueva y coste de materiales (Coulson, $1999)^{4}$.

Las elasticidades precio son positivas y significativas en todas las regiones salvo para la comunidad de Madrid ${ }^{5}$ y en el periodo considerado. En todos los casos, la reacción es elástica, lo que contrasta con resultados anteriores donde se identificaban algunas regiones con elasticidades menores que la unidad. En este trabajo, se analizan periodos en los que se han completado los ciclos de precios y edificación, mientras las investigaciones de referencia estimaban las elasticidades antes de finalizar el ciclo. Esta diferencia es, posiblemente, una de las razones de los resultados más sensibles que aparecen en este trabajo, y acorde con lo que la evidencia internacional refleja. Esta reacción de la promoción de viviendas nuevas ante cambios en los precios parece ser autónoma y reflejaría una elevada sensibilidad ante la señal de mercado, por la

\footnotetext{
${ }^{3}$ Se ha estimado también el modelo con mínimos cuadrados generalizados (Pool EGLS) con ponderación de los parámetros de corte transversal en ambos casos. Los resultados son muy similares y no hay cambios sustanciales en su valor o signo. Por ello, se selecciona el modelo más simple e intuitivo para ser presentado en este trabajo.

${ }^{4}$ Este es un resultado sistemático en los modelos de oferta nueva y refleja la endogeneidad entre construcción y costes de materiales: a mayor construcción, aumento de la demanda de materiales y tensión en sus costes. Es un resultado que se corrige al utilizar métodos de estimación con instrumentos o EGLS. Aplicando esta última técnica en este trabajo, los parámetros resultantes no cambian sustancialmente a los aquí aportados, tampoco su significación ni signo. Esto implica que no se ha podido corregir esta relación. No obstante, la similitud en los valores de los parámetros sistemáticamente obtenidos nos da confianza en la robustez de los mismos. Los resultados están disponibles si se solicitan.

${ }^{5}$ Resultado también obtenido en TALTAVULL (2014) y TALTAVULL y GABRIELLI (2015).
} 
que la mayor parte de los promotores en España toman sus decisiones de aumentar/ reducir la edificación ante los cambios en los precios. Estos datos explicarían la rápida reacción de la promoción ante el inicio de la caída de los precios en España, con fuertes contracciones de las viviendas iniciadas en prácticamente todos los territorios, como se vería en el Gráfico 2.

El número de parcelas urbanas, variable que aproxima el efecto del suelo, aparece con efectos significativos en la edificación en seis de las dieciséis CCAA analizadas, en Castilla La Mancha y Ceuta y Melilla son significativas al 5 por 100, mientras que en Castilla y León, Extremadura, Madrid y la Rioja, lo son al 1 por 100. En todas ellas, la elasticidad es positiva y muy elevada, mostrando una reacción elástica de la construcción ante cambios en la oferta de suelo, lo que sugiere que la edificación crece más que proporcionalmente ante la aparición de nuevas parcelas disponibles para construir en estas regiones. Este resultado parece reflejar una fuerte dependencia del suelo en el proceso de nueva construcción de estas regiones, sugiriendo que la reacción al resultado de planes nuevos de ordenación ha sido (en el periodo analizado) inmediata con fuertes procesos edificadores. Este resultado debe ser interpretado con cuidado dada las distintas características de las regiones en las que se da. Por ejemplo, en el caso de Castilla-la Mancha y Madrid, la fortaleza de la demanda (del segundo) justificaría la reacción capturada por el modelo: con presión de demanda persistente, cualquier disponibilidad de suelo hace crecer la edificación. El caso de Ceuta y Melilla es similar, aunque acrecentado por la escasez endémica de suelo y la fuerte presión de demanda en los mercados de ambas ciudades. Sin embargo, Castilla y León y Extremadura han sido las regiones con menores presiones de demanda durante todo el periodo. En trabajos previos se ponía de manifiesto una baja significatividad de la elasticidad precio de oferta nueva en ambas regiones indicando limitada sensibilidad. Este modelo identifica ahora ambas sensibilidades por separado (a precios y a la oferta sobrerreacción de los promotores cuando hay disponibilidad de suelo independientemente del resto de los determinantes de edificación (precios, costes y otras variables).

Es un resultado remarcable el que las CCAA donde se ha experimentado los mayores ciclos de edificación, no presenten dependencia del aumento del suelo disponible, como son los casos de la Comunidad Valenciana, Cataluña o Andalucía. O tampoco en aquellas donde la demanda internacional ha liderado los ciclos, como en los archipiélagos españoles. Este resultado sugiere que en ninguna de esas regiones la oferta de suelo ha sido un factor determinante de la toma de decisiones de edificación.

Este ejercicio puede aportar alguna explicación adicional a la forma en la que la disponibilidad de suelo ha afectado al equilibrio en los mercados de oferta nueva de viviendas en España. Tradicionalmente, se ha entendido que los mecanismos institucionales para dotar suelo en el mercado dependían de la regulación existente y de la disponibilidad física de suelo, por lo que los efectos fijos (que se interpretan como un indicador que captura los efectos de las características inobservadas de las regiones sobre el modelo de construcción de vivienda nueva) del modelo deberían 
medir este impacto. Estos efectos cambian cuando se incorpora explícitamente la oferta de suelo como variable independiente en el modelo (extrayendo su impacto sobre los efectos fijos). El Gráfico 3 representa los valores normalizados de los efectos fijos de las regiones en un caso y en otro. En la mayor parte de las CCAA, los efectos fijos en los dos modelos presentan el mismo signo, y los que acumulan el efecto de la oferta de suelo (que luego eliminan cuando se externaliza esta variable en el modelo) serían Andalucía, Aragón, Cataluña y Murcia. Hay un segundo grupo de CCAA donde los efectos fijos 'sin oferta de suelo' presentan un valor mayor indicando que sus componentes regionales inobservados afectan en mayor medida la edificación final que en el caso contrario, sugiriendo un efecto de las regulaciones sobre la edificación mayor que de la disponibilidad efectiva de suelo. Estos serían Asturias, Baleares, Canarias, Castilla La Mancha, Comunidad Valenciana y Galicia. Esta diferencia puede ser interpretada por la existencia de condicionantes de la edificación que aparecen una vez la oferta de suelo se ha concretado.

\section{GRÁFICO 3}

EFECTOS FIJOS REGIONALES SOBRE EL MODELO DE OFERTA DE VIVIENDAS NUEVAS

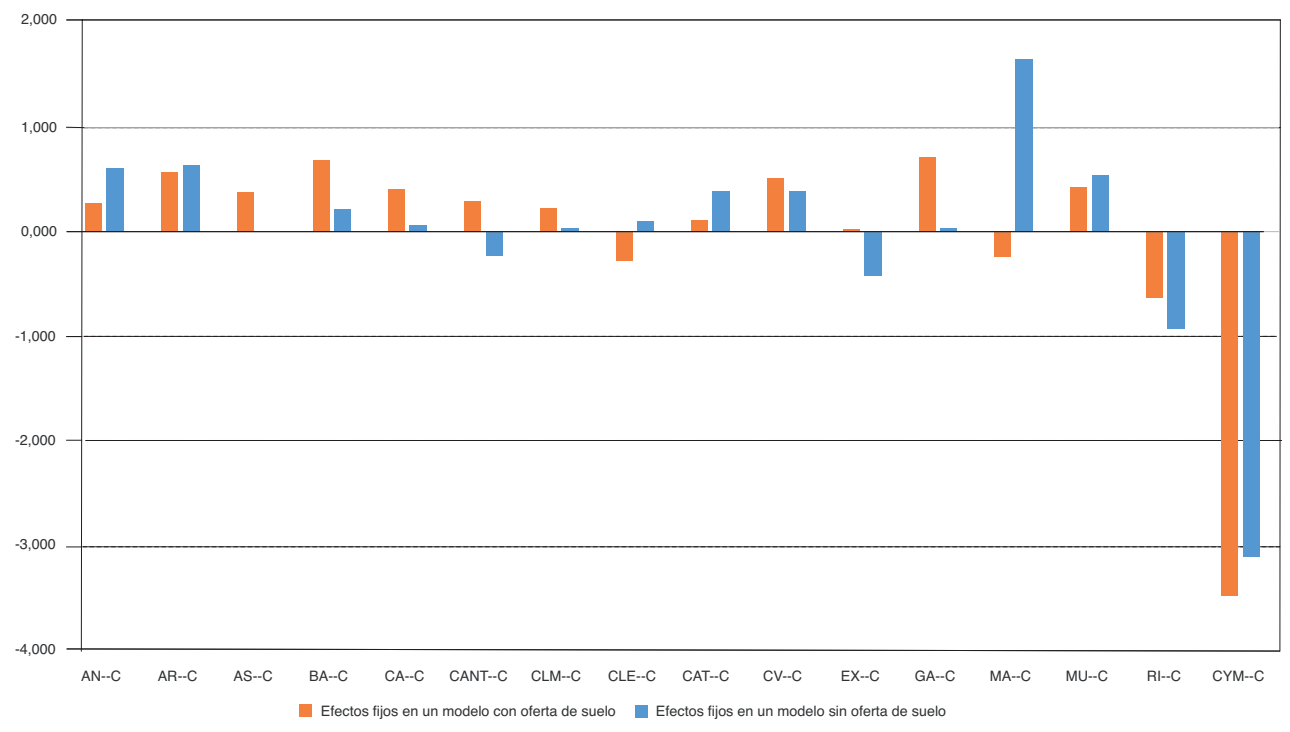

FUENTE: Elaboración propia. 
Dos de las regiones presentan efectos fijos negativos, la Rioja y Ceuta y Melilla, la primera pudiéndose clasificar en el último grupo antes mencionado (es decir, la individualización de la oferta de suelo aumenta el efecto de los componentes inobservables de la región sobre la edificación de viviendas); los efectos fijos de las ciudades autónomas muestran como el efecto regional sobre la edificación se reduce cuando la oferta de suelo se incluye de forma independiente en el modelo. En los dos casos, la elasticidad de respuesta de las viviendas iniciadas ante cambios en la oferta de suelo es significativa (es decir, actúan reduciendo el ritmo de edificación).

Cantabria, Castilla y León, Extremadura y Madrid cambian el signo de sus efectos fijos cuando se toma la oferta de suelo de manera independiente. Los valores para Madrid y Castilla y León sugieren gran relevancia de la oferta de suelo como indicador de las características regionales, de manera que, individualizándola, prácticamente capturan todos los efectos específicos que pueda tener la región en este modelo. Si el argumento es válido, este resultado sugiere que el mercado del suelo es uno de los más importantes para justificar el proceso de edificación (y no tanto la regulación, el sistema de permisos o los potenciales poderes de mercado del conglomerado edificador). En Cantabria y Extremadura ocurre lo contrario, y la individualización del papel de la oferta del suelo parece hacer aflorar otras características regionales con efectos sobre el proceso edificador.

Otra cuestión relevante que aparece con la interpretación de los resultados resulta en la evaluación sobre cómo la sensibilidad precio de los promotores podría cambiar en presencia de información sobre la oferta de suelo. El Gráfico 4 representa los valores de las elasticidades de los dos modelos (el completo y el restringido sin oferta de suelo). Se esperaría que la sensibilidad de los promotores ante cambios en los precios sea mayor si se posee más información y, especialmente, información sobre la disponibilidad de suelo; es decir, la sensibilidad-precio del promotor de viviendas es mayor si conoce la oferta de suelo. Como la mayoría de estas regiones no presentan una elasticidad oferta de suelo estadísticamente significativa, se podría inferir que (en esos casos) la reacción ante variaciones en la oferta de suelo se acumula a la de los cambios en los precios.

Se podría esperar que las elasticidades obtenidas en el modelo completo fuesen mayores que las del modelo restringido (donde la información del suelo no es explícita y se entremezcla con otras para afectar a la oferta). Esta intuición se ve respaldada por los resultados que se presentan en el Gráfico 4, donde la mayor parte de las regiones exhibe una elasticidad en el modelo completo superior o igual a la del modelo restringido, respaldando la idea de que la sensibilidad ante cambios en los precios es mayor con información sobre disponibilidad de suelo, o bien que la información sobre disponibilidad de suelo se traslada a la toma de decisiones vía precios. No ocurre así (con una pequeña diferencia) en Aragón, Baleares, la Rioja, y (con una gran diferencia), en Ceuta y Melilla. La interpretación de este resultado pondría el acento en la sensibilidad de los promotores ante cambios de los precios que puede deberse a su reacción ante cambios en la oferta de suelo. En los casos de las dos Castillas, los resultados sugieren que la información sobre el suelo ejercería 


\section{GRÁFICO 4}

\section{SENSIBILIDAD PRECIO Y SUELO EN LA OFERTA DE VIVIENDAS NUEVAS}

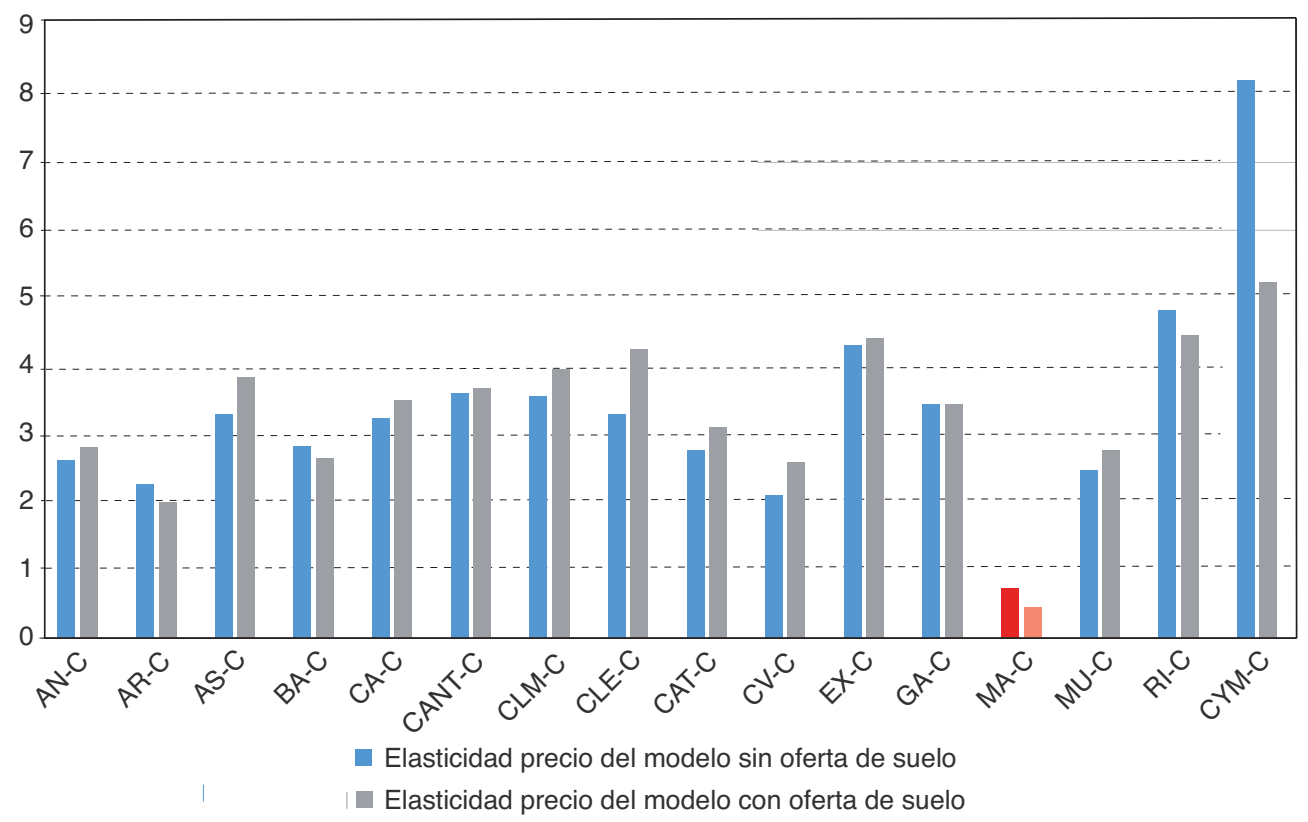

FUENTE: Elaboración propia.

una presión positiva sobre la edificación: desde los precios y desde la oferta, a modo de un mecanismo acelerador de la edificación.

\section{Conclusiones}

En este trabajo se añade evidencia a la literatura sobre la oferta de viviendas nuevas en España. Se estima una nueva ecuación de oferta en el mercado residencial español en su conjunto y en sus regiones, mostrando cómo los promotores reaccionan de forma elástica ante cambios en los precios y respaldando, así, una explicación que justifique la fuerte contracción del ciclo de edificación a partir de 2007. Se construye un pseudo-panel con datos agregados de estadísticas de vivienda por regiones, con base trimestral, desde 1990 a 2015 procedentes de estadísticas oficiales, incluyendo un indicador de disponibilidad de suelo medido a través de información catastral. La ecuación de la oferta de viviendas nuevas se estima usando métodos de panel con un estimador de efectos fijos que permiten capturar la heterogeneidad regional. El modelo se controla por cambios estructurales. La capacidad explicativa de los cambios en la edificación del modelo es elevada (más de un 91 por 100) y se estiman elasticidades-precio altas para todas las regiones salvo Madrid, y elasticidades-oferta de suelo solo significativas para seis regiones, sugiriendo que estas CCAA son muy 
sensibles a los cambios en la oferta de suelo pudiendo, como en los casos de ambas Castillas, generarse crecimientos acumulados en la edificación derivados de esa alta sensibilidad. Las elevadas elasticidades justificarían que el proceso de ajuste después del shock de 2007 haya sido más fuerte en las cantidades que en los precios, dando una explicación a la fuerte reducción del ciclo de edificación.

Los resultados por regiones apoyan esta última interpretación, con especial efecto por periodos, primero en regiones de la costa mediterránea (en 2007) y luego en áreas del interior (como Madrid o Rioja en 2010).

Quedan muchas incógnitas para llegar identificar con mayor precisión las decisiones de los promotores que tanto efecto agregado tiene para la economía, lo que implica un área de investigación por completar en este sector. 


\section{ANEXO}

\section{CUADROS}

\section{CUADRO 1}

BASE DE DATOS DE OFERTA DE VIVIENDAS NUEVAS. ESTADÍSTICAS DESCRIPTIVAS*

\begin{tabular}{|l|c|c|c|c|c|c|c|c|c|c|}
\hline \multicolumn{1}{|c|}{$\begin{array}{c}\text { Periodo: 1990Q1 } \\
\text { 2015Q4 }\end{array}$} & Mean & $\begin{array}{c}\text { Me- } \\
\text { dian }\end{array}$ & $\begin{array}{c}\text { Maxi- } \\
\text { mum }\end{array}$ & $\begin{array}{c}\text { Mini- } \\
\text { mum }\end{array}$ & $\begin{array}{c}\text { Std. } \\
\text { Dev. }\end{array}$ & $\begin{array}{c}\text { Skew- } \\
\text { ness }\end{array}$ & $\begin{array}{c}\text { Kur- } \\
\text { tosis }\end{array}$ & N & $\begin{array}{c}\text { Cross } \\
\text { sections }\end{array}$ & Fuente \\
\hline Viviendas iniciadas & 7,673 & 7,840 & 10,729 & 0,000 & 1,482 & $-0,799$ & 4,044 & 1522 & 16 & MFOM \\
\hline Costes de materiales & 4,450 & 4,418 & 4,746 & 4,157 & 0,175 & $-0,110$ & 1,623 & 1522 & 16 & MFOM \\
\hline Costes de mano de obra & 4,300 & 4,333 & 4,660 & 3,704 & 0,275 & $-0,290$ & 1,959 & 1522 & 16 & MFOM \\
\hline Tipos de interés reales & 3,392 & 2,542 & 12,390 & $-1,120$ & 3,035 & 0,896 & 2,949 & 1522 & 16 & BDE \\
\hline $\begin{array}{l}\text { Número de parcelas } \\
\text { urbanas }\end{array}$ & 13,209 & 13,162 & 14,725 & 9,790 & 0,998 & $-1,022$ & 4,655 & 1522 & 16 & Catastro \\
\hline $\begin{array}{l}\text { Precios de las viviendas } \\
\text { reales }\end{array}$ & 7,015 & 7,001 & 7,992 & 6,223 & 0,361 & 0,118 & 2,459 & 1522 & 16 & MFOM \\
\hline
\end{tabular}

NOTA: * Las variables están en términos logarítmicos salvo los tipos de interés reales.

\section{CUADRO 2}

TEST DE RAICES UNITARIAS EN LA POOL DE DATOS

(Series: En logs: Precios reales, viviendas iniciadas y número de parcelas catastrales para 16 regiones, costes de materiales, costes de mano de obra como variables comunes. En niveles, los tipos de interés reales para las 16 regiones.

\section{Periodo: 1990q1-2015q1)}

\begin{tabular}{|l|c|c|c|c|}
\hline \multicolumn{1}{|c|}{ Method } & Statistic & Prob. & Cross-sections & Obs \\
\hline Null: Unit root (assumes common unit root process) & 51 & 4778 \\
\hline Levin, Lin and Chu t* & $-6,95439$ & 0 & 51 & 4778 \\
\hline Null: Unit root (assumes individual unit root process) & 2,08948 & 0,9817 & 51 & 4778 \\
\hline Im, Pesaran and Shin W-stat & 85,7671 & 0,8761 & 51 & 4949 \\
\hline ADF-Fisher Chi-square & 93,5763 & 0,7122 & 5 \\
\hline PP - Fisher Chi-square &
\end{tabular}

NOTAS: Exogenous variables: Individual effects. Newey-West automatic bandwidth selection and Bartlett kernel. 


\section{CUADRO 3}

TEST DE COINTEGRACIÓN RESIDUAL DE PEDRONI

(Series: LRPH, LCM, LCS, RINT, LVIVIN y LPAR, para las 16 regiones. Periodo: 1990q1-2015q1. Sin tendencia determinística asumida, 1 desfase.

\begin{tabular}{|c|c|c|c|c|}
\hline \multicolumn{5}{|c|}{ H alternativa: hay un coeficiente de AR común (within-dimension) } \\
\hline & Statistic & Prob. & $\begin{array}{l}\text { Weighted } \\
\text { Statistic }\end{array}$ & Prob. \\
\hline Panel v-Statistic & $-1,347498$ & 0,9111 & $-1,672672$ & 0,9528 \\
\hline Panel rho-Statistic & 1,898408 & 0,9712 & 1,775067 & 0,9621 \\
\hline Panel PP-Statistic & 0,786916 & 0,7843 & 0,89741 & 0,8152 \\
\hline Panel ADF-Statistic & 3,590558 & 0,9998 & 3,853066 & 0,9999 \\
\hline \multicolumn{5}{|c|}{ H alternativa: Hay un coeficiente AR individual (between-dimension) } \\
\hline & Statistic & Prob. & & \\
\hline Group rho-Statistic & 3,035721 & 0,9988 & & \\
\hline Group PP-Statistic & 1,684532 & 0,954 & & \\
\hline Group ADF-Statistic & 5,144122 & 1 & & \\
\hline \multicolumn{5}{|c|}{ Test de efectos fijos redundantes } \\
\hline Effects Test & Statistic & d.f. & Prob. & \\
\hline Cross-section F & 519,88 & $-15,1338$ & 0.0000 & \\
\hline Cross-section Chi-square & $2.789,40$ & 15 & 0.0000 & \\
\hline
\end{tabular}




\section{CUADRO 4}

\section{RESULTADOS. OLS EN DATOS DE PANEL CON EFECTOS FIJOS}

\begin{tabular}{|c|c|c|c|c|c|c|c|c|}
\hline \multirow{3}{*}{$\begin{array}{c}\text { Variable } \\
\text { depen- } \\
\text { diente }\end{array}$} & \multicolumn{8}{|c|}{ log viviendas iniciadas } \\
\hline & \multicolumn{5}{|c|}{ Modelo 1. Con oferta de suelo } & \multicolumn{3}{|c|}{$\begin{array}{c}\text { Modelo 2. Restringido (sin } \\
\text { oferta de suelo) }\end{array}$} \\
\hline & $\boldsymbol{\beta}_{1}$ & t-stat & & & & $\boldsymbol{\beta}_{\mathbf{1}}$ & t-stat & \\
\hline \multicolumn{9}{|c|}{ Parámetros comunes } \\
\hline $\mathbf{C}$ & $-181,4 * * *$ & $-6,99$ & & & & $-12,47 * * *$ & $-8,46$ & \\
\hline $\log (\mathrm{cm})$ & $0,99 * * *$ & 3,25 & & & & $1,08 * * *$ & 3,58 & \\
\hline $\log (\mathrm{cs})$ & $-9,05 * * *$ & $-22,1$ & & & & $-7,60 * * *$ & $-28,72$ & \\
\hline \multicolumn{9}{|c|}{ Parámetros específicos } \\
\hline & \multicolumn{2}{|c|}{$\log (\mathbf{r p h})$} & \multicolumn{2}{|c|}{$\log (S)$} & $\begin{array}{c}\text { Efectos } \\
\text { fijos } \\
\text { (norma- } \\
\text { lizados) }\end{array}$ & \multicolumn{2}{|c|}{$\log (\mathbf{r p h})$} & $\begin{array}{c}\text { Efectos } \\
\text { fijos } \\
\text { (norma- } \\
\text { lizados) }\end{array}$ \\
\hline & $\beta_{7, \mathrm{k}}$ & $t$-stat & $\beta_{7, \mathrm{k}}$ & $t$-stat & $\beta_{7, \mathrm{k}}$ & $\beta_{7, \mathrm{k}}$ & $t$-stat & $\beta_{7, \mathrm{k}}$ \\
\hline AN & $2,80 * * *$ & 5,47 & 4,16 & 1,27 & 0,274 & $2,61 * * *$ & 4,72 & 0,61 \\
\hline AR & $1,99 * * *$ & 4,02 & 0,82 & 0,28 & 0,569 & $2,26 * * *$ & 4,60 & 0,64 \\
\hline AS & $3,84 * * *$ & 5,33 & 2,52 & 1,82 & 0,379 & $3,29 * * *$ & 4,82 & 0,01 \\
\hline $\mathrm{BA}$ & $2,64 * * *$ & 3,94 & $-1,45$ & $-0,49$ & 0,688 & $2,82 * * *$ & 5,63 & 0,21 \\
\hline $\mathrm{CA}$ & $3,49 * * *$ & 5,46 & 2,23 & 0,81 & 0,412 & $3,24 * * *$ & 4,80 & 0,06 \\
\hline CANT & $3,67 * * *$ & 5,86 & 4,02 & 1,60 & 0,296 & $3,61 * * *$ & 6,94 & $-0,24$ \\
\hline CLM & $3,96 * * *$ & 7,48 & $4,44 * *$ & 2,22 & 0,224 & $3,55 * * *$ & 6,20 & 0,04 \\
\hline CLE & $4,24 * * *$ & 8,00 & $10,50 * * *$ & 4,44 & $-0,28$ & $3,29 * * *$ & 4,94 & 0,10 \\
\hline CAT & $3,11 * * *$ & 5,92 & 3,21 & 0,58 & 0,112 & $2,76 * * *$ & 5,12 & 0,39 \\
\hline $\mathrm{CV}$ & $2,58 * * *$ & 3,83 & 1,07 & 0,23 & 0,517 & $2,11 * * *$ & 2,93 & 0,39 \\
\hline EX & $4,42 * * *$ & 8,09 & $7,13 * * *$ & 3,51 & 0,025 & $4,31 * * *$ & 7,08 & $-0,42$ \\
\hline GA & $3,44 * * *$ & 4,72 & $-1,76$ & $-0,70$ & 0,716 & $3,44 * * *$ & 4,63 & 0,04 \\
\hline MA & 0,45 & 1,54 & $12,76^{* * *}$ & 10,52 & $-0,24$ & 0,74 & 1,50 & 1,66 \\
\hline MU & $2,75^{* * *}$ & 5,06 & 2,37 & 0,87 & 0,429 & $2,47 * * *$ & 4,54 & 0,55 \\
\hline $\mathrm{RI}$ & $4,46 * * *$ & 11,23 & $18,34 * * *$ & 3,65 & $-0,64$ & $4,83 * * *$ & 14,58 & $-0,93$ \\
\hline CYM & $5,24 * * *$ & 2,99 & $73,56 * *$ & 2,22 & $-3,49$ & $8,20 * * *$ & 5,75 & $-3,11$ \\
\hline \multicolumn{2}{|l|}{$\operatorname{Adj}^{2}$} & 0,92 & & & & & 0,917 & \\
\hline \multicolumn{2}{|c|}{$\Sigma \varepsilon^{2}$} & 224 & & & & & 237,08 & \\
\hline \multicolumn{2}{|c|}{ Log likelihood } & -704 & & & & & $-744,6$ & \\
\hline \multicolumn{2}{|c|}{ F-statistic } & $151 * * *$ & & & & & $167,73 * * *$ & \\
\hline \multicolumn{2}{|l|}{ DW } & 1,64 & & & & & 1,6072 & \\
\hline
\end{tabular}

NOTA: Los ajustes de cada modelo incluyen igualmente los parámetros de tipos de interés y los efectos de los shocks sobre las regiones además de la corrección por el proceso autorregresivo. Los tipos de interés reales son significativos solo para la CA de Madrid $\left(\mathrm{G}_{\text {Madrid }}=-0,1163\right)$ y para Rioja $\left(\mathrm{G}_{\text {Rioja }}=-0,094\right)$. El shock de 2007 es significativo solo en la Comunidad Valenciana, Cataluña, Murcia y Rioja, mientras que el de 2010 lo es en Cantabria, Madrid y Ceuta y Melilla. ** p-value $<0,05, * * *$ p-value $>0,01$. 


\section{Referencias bibliográficas}

[1] ARNOTT, R. (1987). «Economic Theory and Housing», en Handbook of Regional and Urban Economics, vol. II, cap. 24, 959-988.

[2] BARKER, K. (2003). Review of Housing Supply. Interim report-Analysis. HM Treasury, Londres.

[3] BLACKLEY, D. M. (1999). «The Long-Run Elasticity of New Housing Supply in the United States: Empirical Evidence for 1950 to 1994». Journal of Real Estate Finance and Economics, 18 (1), 25-42.

[4] BRAMLEY, G. (1993). «Land-Use Planning and the Housing Market in Britain-The Impact on Housebuilding and House Prices». Environment Planning, 25, 1021-1052.

[5] BRAMLEY, G. (2003). «Planning regulation and housing supply in a market system». En T. O’Sullivan y K. Gibb, Housing Economics and Public Policy, Blackwell Publishing, Londres.

[6] COULSON, N. E. (1999). «Housing Inventory and Completion». Journal of Real Estate Finance and Economics, 18 (1), 89-105.

[7] DIPASQUALE, D. (1999). «Why don't we know more about housing supply?». Journal of Real Estate Finance and Economics, 18, 9-23.

[8] DIPASQUALE, D. y WHEATON, W. (1994). «Housing market dynamics and the future of housing prices». Journal of Urban Economics, 35, 1-28.

[9] FERGUS, J. T. (1999). «Where, when and by how much does abnormal weather affect housing construction?». The Journal of Real Estate Finance and Economics, 18 (1), 63-87.

[10] GIBLER, K.; TALTAVULL, P.; CASADO-DÍAZ, J. M.; CASADO-DÍAZ, M y RODRÍGUEZ, V. (2009). «Examining retirement housing preferences among international retiree migrants». International Real Estate Review, 12 (1), 1-22.

[11] GLAESER, E. L.; GYOURKO, J. y SAKS, R. E. (2005). «Urban Growth and Housing Supply». HIER Discussion Paper No. 2.062, SSRN papers series No. 658.343.

[12] GOODMAN, A. C. (2005). «The Other Side of Eight Mile: Suburban Population and Housing Supply». Real Estate Economics, 33 (3), 539-569.

[13] GOODMAN, A. C. y THIBODEAU, T. G. (1998). «Housing Market Segmentation». Journal of Housing Economics, 7, 121-143.

[14] GREEN, R. K. y MALPEZZI, S. (2003). A primer on US Housing Markets and Housing Policy, AREUEA Monograph Series no. 3, Washington.

[15] GREEN, R. K.; MALPEZZI, S. y MAYO, S. (2005). «Metropolitan-specific estimates of the price elasticity of supply of housing and their sources». American Economic Review, 95 (2), 334-339.

[16] GRIMES, A. (2007). Impacts of Land Availability, Housing Supply and Planning Infrastructure on New Zealand House Prices. En Treasury and Reserve Bank of New Zealand conference «The Business Cycle, Housing and the Role of Policy», 10-11, disponible en https://www.researchgate.net/.

[17] GYOURKO, J. y SUMMERS, A. A. (2006). «Residential Land Use Regulation in the Philadelphia MSA». ZEL/Lurie Real Estate Center Working Paper 560, disponible en https://www.researchgate.net/.

[18] GYOURKO, J.; SAIZ, A. y SUMMERS, A. A. (2008). «A new measure of the local regulatory environment for housing markets: The Wharton Residential Land Use Regulatory Index», Urban Studies, 45 (3), 693-729. 
[19] HANUSHEK, E. A. y QUIGLEY, J. M. (1979). «The Dynamics of the Housing Market: A Stock Adjustment Model of Housing Consumption». Journal of Urban Economics, 6 (1), 90-111.

[20] INSTITUTO NACIONAL DE ESTADÍSTICA (INE). Boletín Estadístico. Disponible en $h t t p: / / w w w . i n e . e s$.

[21] MALPEZZI, S. (1996). «Housing Prices, Externalities and Regulation in U.S. Metropolitan Areas». Journal of Housing Research, 7 (2), 209-241.

[22] MALPEZZI, S. y MACLENNAN, D. (2001). «The long-run Price Elasticity of Supply of New Residential Construction in the United States and the United Kingdom». Journal of Housing Economics, 10, 278-306.

[23] MALPEZZI, S. y VANDELL, K. (2002). «Does the low-income housing tax credit increase the supply of housing?». Journal of Housing Economics, 11, 360-380.

[24] MAYER, C. J. y SOMERVILLE, C. T. (2000). «Residential Construction: Using the Urban Growth Model to Estimate Housing Supply». Journal of Urban Economics, 48, 85-109.

[25] MAYO, S. y SHEPPARD, S. (2001). «Housing Supply and the Effects of Stochastic Development Control». Journal of Housing Economics, 10, 109-128.

[26] MEEN, G. (2003). «Housing Cycles and Efficiency». Scottish Journal of Political Economy, 47 (2), 114-140.

[27] MINISTERIO DE FOMENTO. Estadísticas de Edificación y Vivienda. Disponible en http://www.mfom.es.

[28] MUTH, R. y GOODMAN, A. C. (1989). The Economics of Housing markets. Londres, Harwood Academic Publishers.

[29] POTERBA, J. M. (1991). «House Price Dynamics: The Role of Tax Policy and Demography». Brookings Papers on Economic Activity, 2, 143-203.

[30] PRYCE, G. (1999). «Construction Elasticities and Land Availability: a Two-Stage Least-squares Model of Housing Supply Using the Variable Elasticity Approach». Urban Studies, 36 (13), 2283-2304.

[31] QUIGLEY, J. M. (1997). The Economics of Housing. Edward Elgar Publishing, Massachusetts. Vols. 1 y 2.

[32] QUIGLEY, J. M. (2007). «Regulation and Property Values in the United States: The High Cost of Monopoly». Berkeley program on Housing and Urban Policy, WP06-004. Disponible en http://escolarship.org/uc/item/5692w323.

[33] POTERBA, J. M. (1991). «House price dynamics: The role of tax policy and demography». Brookings Papers on Economic Activity, 2, 143-203.

[34] ROSENTHAL, S. (1999). «Residential Buildings and the Cost of Construction: New Evidence on the Efficiency of the Housing Market». The Review of Economics and Statistics, 81 (2), 288-302.

[35] SAIZ, A. (2010). «The geographic determinants of housing supply». The Quarterly Journal of Economics, 125 (3), 1253-1296.

[36] SOMERVILLE, C. T. (1999a). «Residential Construction Costs and the Supply of New Housing: Endogeneity and Bias in Construction Cost Indexes». Journal of Real Estate Finance and Economics, 18 (1), 43-62.

[37] SOMERVILLE, C. T. (1999b). «The Industrial Organization of Housing Supply: Market Activity, Land Supply and the Size of Homebuilders Firms». Real Estate Economics, 27 (4) 669-694. 
[38] TALTAVULL DE LA PAZ, P. (2006). «La oferta de viviendas y el mercado inmobiliario en España». Papeles de Economía Española, 109, 156-181.

[39] TALTAVULL DE LA PAZ, P. (2014). «New housing supply and price reactions: Evidence from Spanish markets». Journal of European Real Estate Research, 7 (1), 4-28.

[40] TALTAVULL DE LA PAZ, P. y GABRIELLI, L. (2015). «Housing Supply and Price Reactions: A Comparison Approach to Spanish and Italian Markets». Housing Studies, 30 (7), 1036-1063.

[41] TOPEL, R. y ROSEN, S. (1988). «Housing investment in the United States». Journal of Political Economy, 96, 718-740. 